\title{
Several classes of single variable polynomials over finite fields
}

\author{
Marie Henderson
}

In this thesis we investigate several well-known classes of polynomials defined over a finite field $\mathbb{F}_{q}$. We consider various properties of the Dickson polynomials, in particular the Dickson polynomials of the second kind, and the linearised and sub-linearised polynomials.

A polynomial which represents a function that permutes the elements of $\mathbb{F}_{q}$ is called a permutation polynomial. We find new classes of permutation polynomials of $\mathbb{F}_{q}$ which are members of the class of Dickson polynomials of the second kind (DPSK). If $a=0$ then the DPSK are given by $f_{k}(X, a)=S^{k}$. The permutation behaviour of these polynomials is well understood so we focus on those where $a \in \mathbb{F}_{q}^{*}$. It is shown that the permutation behaviour of the tow DPSK, $f_{k}(X, a)$ and $f_{k}\left(X, a^{\prime}\right)$ where $a, a^{\prime} \in \mathbb{F}_{q}^{*}$ and $\eta(a)=\eta\left(a^{\prime}\right)$, is equivalent (here $\eta$ represents the quadratic character of $\mathbb{F}_{q}$ ). That is to say, either both polynomials are permutation polynomials of $\mathbb{F}_{q}$ or both polynomials fail to be permutation polynomials of $\mathbb{F}-q$. Various classes dependent on $k, q$ and whether $a \in \mathbb{F}_{q}^{*}$ is a square or non-square in $\mathbb{F}_{q}$ are given. Simple restrictions on those $k$ for which the Dickson polynomial of the second kind $f_{k}(X, a)$ is a permutation polynomial of $\mathbb{F}_{q}$ are obtained.

The composition behaviour of the linearised and sub-linearised polynomials is discussed. A connection between the composition behaviour of certain linearised and sub-linearised polynomials is established. The question of which linearised and sublinearised polynomials are indecomposable over $\mathbb{F}_{q}$ is examined. An application of these results to cryptography is then developed. Cryptosystems based on both the linearised and sub-linearised polynomials are introduced. The security of the systems is discussed briefly.

School of Information Technology

The University of Queensland

Queensland 4072

Australia

e-mail: marie@it.uq.edua.u

Received 15th October, 1997

Thesis submitted to The University of Queensland, March 1997. Degree approved, July 1997. Supervisor: Dr. R. Matthews, Dr. K. Matthews and Dr G. Havas.

Copyright Clearance Centre, Inc. Serial-fee code: 0004-9729/98 \$A2.00+0.00. 